Commun. Fac. Sci. Univ. Ank. Series $A_{1}$ V. 38, pp 15-20 (1988)

\title{
A CLASS OF NONLINEAR SECOND ORDER DIFFERENTIAL EOUATION SOLVABLE BY MEANS OF A LINEAR DIFFERENTIAL EOUATION
}

\author{
CEVAT KART \\ Ankara University Faculty of Science Mathematics Department 06100 Beşevler-ANKARA
}

\section{ABSTRACT}

The solutions of the differential equation $2 \mathrm{yy}^{\prime \prime}=\mathrm{y}^{\prime 2}-4 \mathrm{q}(\mathrm{x}) \mathrm{y}^{2}+\mathrm{c}$ are expressed in terms of solutions of the equation $\mathrm{u}^{\prime \prime}+\mathrm{q}(\mathrm{x}) \mathrm{u}=0$ for different values of the constant $c$.

\section{INTRODUCTION}

Mathematical investigations of natural phenomena generally lead to nonlinear differential equations*. If most of these phenomena are not understood at present, the reason mainly lies in the fact that general methods for solving nonlinear differential equations are not known yet. For this reason it is important to establish some connections between linear and nonlinear differential equations.

It is well known that any homogenous linear differential equation of the second order can be reduced to the canonical form

$$
\begin{aligned}
& u^{\prime \prime}+q(x) u=0 \\
& \text { If } u_{1}, u_{2} \text { are solutions of }(1) \text {, then } \\
& y=u_{1} u_{2}
\end{aligned}
$$

satisfies the nonlinear differential equation

$$
2 \mathrm{yy}^{\prime \prime}=\mathrm{y}^{\prime 2}-4 \mathrm{q}(\mathrm{x}) \mathrm{y}^{2}+\mathrm{c}
$$

for certain values of the constant c. In fact, substituting (2) and its derivatives in (3) we have

$$
\left(\mathbf{u}_{1} \mathbf{u}_{2}{ }^{\prime}-\mathbf{u}_{1}{ }^{\prime} \mathbf{u}_{2}\right)^{2}+\mathbf{c}=0 .
$$

* Concerning the theory of nonlinear differential equations. See, for instance [1], [2], [3] and [4].

ISSN 02571-081 A.Ü. Basımevi 
Hence (2) is a solution to (3) if and only if

$$
\mathrm{c}=-\mathbf{W}^{2}{ }_{\mathrm{o}}
$$

with $\mathbb{W}\left(\mathrm{u}_{1}, \mathrm{u}_{2}\right)$ denoting the Wronskian of $\mathrm{u}_{1}, \mathrm{u}_{2}$ :

$$
\mathbb{W}\left(\mathbf{u}_{1}, \mathbf{u}_{2}\right)=\mathbf{u}_{1} \mathbf{u}^{\prime}{ }_{2}-\mathbf{u}^{\prime}{ }_{1} \mathbf{u}_{2}=\mathbb{W}_{\mathbf{0}} .
$$

The purpose of the present work is to investigate the two-parameter solutions of (3) for different values of $c$, with the assumption that $q$ is real-valued.

\section{SOLUTIONS OF EQUATION (3) FOR DIFFERENT VALUES OF THE CONSTANT c.}

Case I: $c=0$

Let $u=c_{1} u_{2}+c_{2} u_{2}$ be the general solution of $(1)$. Then $u$ and $u$ are (linearly dependent) solutions and $W_{1}(u, u)=\left(c_{1} c_{2}-c_{1} c_{2}\right) W_{0}=0$ so that the two-parameter solution of (3) reads

$$
\mathbf{y}=\mathbf{u} \cdot \mathbf{u}=\mathbf{u}^{2}=\left(\mathbf{c}_{1} \mathbf{u}_{1}+\mathbf{c}_{2} \mathbf{u}_{2}\right)^{2} .
$$

In fact, the transformation $y=u^{2}$ in (1) at once gives

$$
2 \mathrm{yy}^{\prime \prime}=\mathrm{y}^{\prime 2}-4 \mathrm{q}(\mathrm{x}) \mathrm{y}^{2} .
$$

Case II: $\mathrm{c}<0$ and $\mathrm{c}=-\mathrm{W}^{2}{ }_{0}$

The solutions of Eq.(3) are of the form

$$
\mathbf{y}=\left(\mathbf{a}_{1} \mathbf{u}_{1}+\mathbf{a}_{2} \mathbf{u}_{2}\right)\left(\mathbf{b}_{1} \mathbf{u}_{1}+\mathbf{b}_{2} \mathbf{u}_{2}\right)
$$

where $a_{1}, a_{2}, b_{1}, b_{2}$ are arbitrary (not necessarily independent) constants. The factors occurring in (8) are linearly independent if and only if

$$
a_{1} b_{2}-a_{2} b_{1} \neq 0 .
$$

By (9), $a_{1} \neq 0$ or $a_{2} \neq 0$ so that we can set $a_{1}=1$ without loss of generality. Then (8) takes the form

$$
\mathbf{y}=\left(\mathbf{u}_{1}+\mathbf{c}_{1} \mathbf{u}_{2}\right)\left(\mathbf{c}_{2} \mathbf{u}_{1}+\mathbf{c}_{3} \mathbf{u}_{2}\right) .
$$

Then Wronskian of $u_{1}+c_{1} u_{2}$ and $c_{2} u_{1}+c_{3} u_{2}$ is

$$
W_{2}=\left(c_{3}-c_{1} c_{2}\right) W_{0} .
$$


It then follows that the expression (10) satisfies (3) if

$$
\mathrm{W}_{2}^{2}+\mathrm{c}=0
$$

From (11) and (12) we have

$$
\mathrm{W}_{2}^{2}=-\left(\mathrm{c}_{3}-\mathrm{c}_{1} \mathrm{c}_{2}\right)^{2} \mathrm{c}=-\mathrm{c}
$$

and so

$$
c_{3}=c_{1} c_{2} \pm 1=k_{1} .
$$

Accordinly, the two-parameter solution of (3) is of the form

$$
\mathrm{y}=\mathrm{c}_{2} \mathrm{u}^{2}{ }_{1}+\left(\mathrm{k}_{1}+\mathrm{c}_{1} \mathrm{c}_{2}\right) \mathrm{u}_{1} \mathrm{u}_{2}+\mathrm{c}_{1} \mathrm{k}_{1} \mathrm{u}^{2}{ }_{2}
$$

Case III: $c<0$ and $c \neq-\mathrm{W}^{2}{ }_{0}$

In this case (12) becomes

$$
\mathrm{W}_{2}^{2}=\left(\mathrm{c}_{3}-\mathbf{c}_{1} \mathbf{c}_{2}\right)^{2} \mathrm{~W}_{\mathrm{o}}^{2}=-\mathbf{c} \text {. }
$$

This gives

$$
\mathrm{c}_{3}=\mathrm{c}_{1} \mathrm{c}_{2} \pm \frac{1}{\mathrm{~W}_{\mathrm{o}}} \sqrt{-\mathrm{c}}=\mathrm{k}_{2} \text {. }
$$

Now the two-parameter solution of (3) can be expressed as

$$
\mathbf{y}=\mathbf{c}_{2} \mathbf{u}_{1}{ }^{2}+\left(\mathbf{k}_{2}+\mathbf{c}_{1} \mathbf{c}_{2}\right) \mathbf{u}_{1} \mathbf{u}_{2}+\mathbf{c}_{1} \mathbf{k}_{2} \mathbf{u}_{2}{ }^{2}
$$

Moreover, let

$$
\mathrm{v}_{1}=\alpha \mathbf{u}_{1}, \mathbf{v}_{2}=\beta \mathbf{u}_{2} \quad|\alpha|+|\beta| \neq 0 .
$$

One can choose $\alpha, \beta$ such that $\mathrm{W}\left(\mathrm{v}_{1}, \mathrm{v}_{2}\right)=1$. With this choice $c_{3}$ becomes

$$
\mathbf{c}_{3}=\mathbf{c}_{1} \mathbf{c}_{2} \pm \overline{\mathbf{C}^{\prime} \mathbf{c}}=\mathbf{k}_{3},
$$

and the two-parameter solution of (3) takes the from

$$
\mathrm{y}=\mathrm{c}_{2} \mathbf{v}_{1}{ }_{1}+\left(\mathrm{k}_{3}+\mathrm{c}_{1} \mathrm{c}_{2}\right) \mathrm{v}_{1} \mathbf{v}_{2}+\mathrm{c}_{1} \mathbf{k}_{3} \mathbf{v}_{2}{ }^{2} \text {. }
$$

Case IV: $\mathbf{c}>0$

Let us examine if Eq. (3) may admit of real-valued solutions for c $>0$. By (11), we now have

$$
\mathrm{W}_{2}^{2}=\left(\mathrm{c}_{3}-\mathrm{c}_{1} \mathrm{c}_{2}\right)^{2} \mathrm{~W}_{\mathrm{o}}^{2}=-\mathrm{c}, \mathrm{c}>0
$$


This gives

$$
c_{3}=c_{1} c_{2} \pm \frac{1}{W_{0}} \sqrt{-c}=c_{1} c_{2} \pm \frac{1}{W_{0}} i \sqrt{c}=k_{4} .
$$

Accordinly, the two-parameter (complex) solution of (3) is

$$
\mathbf{y}=\mathbf{c}_{2} \mathbf{u}_{1}{ }_{1}+\left(\mathrm{k}_{4}+\mathrm{c}_{1} \mathbf{c}_{2}\right) \mathbf{u}_{1} \mathbf{u}_{2}+\mathrm{c}_{1} \mathbf{k}_{4} \mathbf{u}_{2}{ }_{2} .
$$

Putting $c_{1}=\alpha_{1}+i \beta_{1}, c_{2}=\alpha_{2}+i \beta_{2},\left(\alpha_{1}, \beta_{1} ; \alpha_{2}, \beta_{2}\right.$ real $)$ we may write (21) as

$$
\begin{aligned}
& y=\alpha_{2} u^{2}{ }_{1}+2\left(\alpha_{1} \alpha_{2}-\beta_{1} \beta_{2}\right) u_{1} u_{2}+\left[\alpha_{2}\left(\alpha_{1}^{2}-\beta_{1}{ }_{1}\right)-\beta_{1}\left(2 \alpha_{1} \beta_{2} \pm \frac{\sqrt{\mathrm{c}}}{\mathbb{W}_{0}}\right)\right] \mathbf{u}^{2} \\
& +\mathbf{i}\left\{\beta_{2} \mathbf{u}_{1}{ }^{2}+\left[2\left(\alpha_{1} \beta_{2}+\alpha_{2} \beta_{1}\right) \pm \frac{\sqrt{\mathbf{c}}}{\mathbf{W}_{0}}\right] \mathbf{u}_{1} \mathbf{u}_{2}+\left[\beta_{2}\left(\alpha^{2}{ }_{1}-\beta^{2}{ }_{1}\right)+\right.\right. \\
& \left.\left.\alpha_{1}\left(2 \alpha_{2} \beta_{1} \pm \frac{\sqrt{\mathrm{c}}}{\mathrm{W}_{\mathrm{o}}}\right)\right] \mathrm{u}_{2}^{2}\right\}
\end{aligned}
$$

The vanishing of the imaginary part in the above equality yields

$$
\beta_{2}=0,2 \alpha_{2} \beta_{1} \pm \frac{1}{\mathbf{W}_{0}} \sqrt{\mathbf{c}}=0 .
$$

This implies

$$
\alpha_{1} \text { arbitrary, } \beta_{1}= \pm \frac{\overline{\sqrt{c}}}{2 \alpha_{2} \bar{W}_{0}}, \alpha_{2} \neq 0, \beta_{2}=0 .
$$

If (23) is satisfied, the solution is real, and can be expressed as

$$
\mathbf{y}=\mathbf{a}_{2} \mathbf{u}_{1}{ }^{2}+2 \alpha_{1} \alpha_{2} \mathbf{u}_{1} \mathbf{u}_{2}+\left(\alpha_{1}^{2} \alpha_{2}+\frac{\mathbf{c}}{4 \alpha_{2} \mathbf{W}_{0^{2}}}\right) \mathbf{u}_{2}{ }^{2}
$$

Case V: Im $\mathbf{c} \neq 0$

If y is real, Eq. (3) implies Imc $=0$. Hence Eq. (3) has no real valued solution for Ime $\neq 0$.

\section{EXAMPLES}

Example 1. $2 \mathrm{yy}^{\prime \prime}=\mathrm{y}^{\prime 2}-(\mathrm{x}+1)^{-2} \mathrm{y}^{2}-1$.

This equation is of the type (3) with $q(x)=\frac{1}{4}(x+1)^{-2}, c=$ - 1. The associated linear equation reads

$$
\mathbf{u}^{\prime \prime}+\frac{1}{4}(x+1)^{-2} \mathbf{u}=0,
$$


with the linearly independent solutions

$$
\mathrm{u}_{1}=(\mathrm{x}+1)^{\frac{1}{2}}, \quad \mathrm{u}_{2}=(\mathrm{x}+1)^{\frac{1}{2}} \ln (\mathrm{x}+\mathrm{-1}) .
$$

Presently $W_{0}=1$, and so $W_{0}=-c . B y(14)$ the real -valued solution is

$$
y=c_{2}(x+1)+\left(k_{1}+c_{1} c_{2}\right)(x+1) \ln (x+1)+c_{1} k_{1}(x+1) l n^{2}(x+1) .
$$

Example 2. $2 \mathrm{yy}^{\prime \prime}=\mathrm{y}^{\prime 2}+8 \mathrm{x}^{-2} \mathrm{y}^{2}+\mathrm{c}$.

Comparison of (28) with (3) yields $q(x)=-2 x^{-2}$. The corresponding linear equation is

$$
\mathbf{u}^{\prime \prime}-2 \mathbf{x}^{-2} \mathbf{u}=0 .
$$

It has linearly independent solutions

$$
\mathbf{u}_{1}=\mathrm{x}^{-1}, \quad \mathbf{u}_{2}=\mathrm{x}^{2}
$$

with the Wronskian $\mathrm{W}_{0}=3$.

(i) If $\mathrm{c}=\mathbf{0}$ the solution is, by (6),

$$
y=\left(c_{1} x^{-1}+c_{2} x^{2}\right)^{2}, \quad x \neq 0
$$

(ii) If $c=-9$ we have $W_{0^{2}}=-c$. By (14) the solution is of the form

$$
\mathrm{y}=\mathrm{c}_{2} \mathrm{x}^{-2}+\left(\mathrm{k}_{1}+\mathrm{c}_{1} \mathrm{c}_{2}\right) \mathrm{x}+\mathrm{c}_{1} \mathbf{k}_{1} \mathrm{x}^{4}, \mathrm{x} \neq 0 .
$$

(iii) If $\mathrm{c}<0$ and $\mathrm{c} \neq-9$, then by (16) the solution reads $\mathrm{y}=\mathrm{c}_{2} \mathrm{x}^{-2}$ $+\left(\mathbf{k}_{2}+\mathbf{c}_{1} \mathbf{c}_{2}\right) \mathbf{x}+\mathbf{c}_{1} \mathbf{k}_{2} \mathbf{x}^{4}, \mathbf{x} \neq 0$.

(iv) If $\mathrm{c}>0$, by (24) the solution becomes

$$
\mathrm{y}=\alpha_{2} \mathrm{x}^{-2}+2 \alpha_{1} \alpha_{2} \mathrm{x}+\left(\alpha_{1}{ }^{2} \alpha_{2}+\frac{\mathbf{c}}{36 \alpha_{2}}\right) \mathrm{x}^{4} .
$$

Example 3. $2 \mathrm{yy}^{\prime \prime}=\mathrm{y}^{\prime 2}+\left(4 \mathrm{e}^{2 \mathrm{x}}+1\right) \mathrm{y}^{2}+\mathrm{c}, \quad \mathrm{c} \leq 0$.

In the present example, $q(x)=-\left(e^{2 x}+\frac{1}{4}\right)$, and so the associated linear equation is

$$
u^{\prime \prime}-\left(e^{2 x}+\frac{1}{4}\right) u=0 .
$$


Putting $\mathrm{z}=\mathrm{e}^{\mathrm{x}}$, this equation becomes

$$
\frac{\mathrm{d}^{2} \mathbf{u}}{\mathrm{d} \mathrm{z}^{2}}+\frac{1}{2} \frac{\mathrm{du}}{\mathrm{dz}}-\left(1+\frac{1}{4 \mathrm{z}^{2}}\right) \mathrm{u}=0 .
$$

The change $\mathrm{u}=\mathrm{z}^{-1 / 2} \mathrm{v}$ transforms (32) into

$$
\frac{\mathbf{d}^{2} \mathbf{v}}{\mathbf{d z}^{2}}-\mathbf{v}=0 \text {. }
$$

From the above relations it is easily found that

$$
\mathrm{u}_{1}=\mathrm{e}^{-\left(\frac{1}{2} \mathrm{x}+\mathrm{e}^{\mathrm{x}}\right)}, \mathrm{u}_{2}=\mathrm{e}^{-\left(\frac{1}{2} \mathrm{x}-\mathrm{e}^{\mathrm{x}}\right)} .
$$

The Wronskian of $u_{1}, u_{2}$ is $W_{0}=2$.

(i) $c=-4$. Presently $c=-W_{0^{2}}$ so that by (14) the solution of $(30)$ is

$$
\mathrm{y}=\mathrm{c}_{2} \mathrm{e}^{-\left(\mathrm{x}+2 \mathrm{e}^{\mathrm{x}}\right)}+\left(\mathrm{k}_{1}+\mathrm{c}_{1} \mathrm{c}_{2}\right) \mathrm{e}^{-\mathrm{x}}+\mathrm{c}_{1} \mathrm{k}_{1} \mathrm{e}^{-\left(\mathrm{x}-2 \mathrm{e}^{\mathrm{x}}\right)} .
$$

(ii) $\mathrm{c}<0, \mathrm{c} \neq-4$. In this case, by (16) the solution of (30) is of the from

$$
y=c_{2} e^{-\left(x+2 e^{x}\right)}+\left(k_{2}+c_{1} c_{2}\right) e^{-x}+c_{1} k_{2} e^{-\left(x-2 e^{x}\right)} .
$$

[1] BIRKHOFF, G. and ROTA, G.C., Ordinary Differential Equationc, 2nd ed. Blaisdell Pub. Co., Waltham, Mass., 1968.

[2] DAVIES, HAROLD T., Introduction to Nonlinear Differential and Integral Equations, Dover Pub., Inc., New York, 1962.

[3] HERBST, R.T., The Equivalence of Linear and Nonlinear Differential Equations, Proc. Amer. Math. Soc. 76, 1956.

[4] AMES, W.F., Nonlinear Ordinary Differential Equations in Transport Process, Academic Press, New York, 1968. 